Pacific Journal of Mathematic 


\title{
A RANDOM FIXED POINT THEOREM FOR A MULTIVALUED CONTRACTION MAPPING
}

\author{
SHIGERU ITOH
}

\begin{abstract}
Some results on measurability of multivalued mappings are given. Then using them, the following random fixed point theorem is proved: Theorem. Let $X$ be a Polish space, $(T, \mathscr{A})$ a measurable space. Let $F: T \times X \rightarrow C B(X)$ be a mapping such that for each $x \in X, F(\cdot, x)$ is measurable and for each $t \in T$, $F(t, \cdot)$ is $k(t)$-contraction, where $k: T \rightarrow[0,1)$ is measurable. Then there exists a measurable mapping $u: T \rightarrow X$ such that for every $t \in T, u(t) \in F(t, u(t))$.
\end{abstract}

1. Introduction. Random fixed point theorems for contraction mappings in Polish spaces were proved by Špaček [8], Hanš [2, 3], etc. For a brief survey of them and related results, we refer the reader to Bharucha-Reid [1, Chapter 3]. On the other hand, fixed point theorems for multivalued contraction mappings in complete metric spaces were obtained by Nadler [7], etc.

In this paper, in $\$ 3$ we give some results on measurability and measurable selectors of multivalued mappings. Then in $\S 4$, using them we prove a random fixed point theorem for a multivalued contraction mapping in a Polish space.

The author wishes to express his gratitude to Professors H. Umegaki and $\mathrm{W}$. Takahashi for their encouragement in preparing this paper.

2. Preliminaries. Throughout this paper, let $(X, d)$ be a Polish space, i.e., a separable complete metric space, and $(T, \mathscr{A})$ a measurable space. For any $x \in X, B \subset X$, we denote $d(x, B)=$ $\inf \{d(x, y): y \in B\}$. Let $2^{X}$ be the family of all subsets of $X, C B(X)$ the family of all nonempty bounded closed subsets of $X, \mathscr{B}$ the $\sigma$-algebra of Borel subsets of $X$, respectively. Let $D$ be the Hausdorff metric on $C B(X)$ induced by $d$. A mapping $S: X \rightarrow C B(X)$ is called $k$-Lipschitz, where $k \geqq 0$, if for every $x, y \in X, D(S(x), S(y)) \leqq k d(x, y)$. When $k<1$, then $S$ is called $k$-contraction. A mapping $F: T \rightarrow 2^{X}$ is called $\left(\mathscr{A}\right.$-) measurable if for any open subset $B$ of $X, F^{-1}(B) \in \mathscr{A}$, where $F^{-1}(B)=\{t \in T: F(t) \cap B \neq \varnothing\}$. Notice that in Himmelberg [5] this is called weakly measurable, but in this paper we use only this type of measurability for multivalued mappings, hence we omit the term 'weakly' for the sake of simplicity. A mapping $u: T \rightarrow X$ is said to be a measurable selector of a measurable mapping $F: T \rightarrow 2^{X}$ if $u$ is measurable and for any $t \in T, u(t) \in F(t)$. 
3. Some results on measurability. In this section we give important results related to the concept of measurability and measurable selector. They play a crucial role in proving a random fixed point theorem in $\S 4$.

Proposition 1. Let $\left\{F_{n}\right\}$ be a sequence of measurable mappings $F_{n}: T \rightarrow C B(X)$, and $F: T \rightarrow C B(X)$ a mapping such that for each $t \in T$, $D\left(F_{n}(t), F(t)\right) \rightarrow 0$ as $n \rightarrow \infty$. Then $F$ is measurable.

Proof. By Himmelberg [5, Theorem 3.5], it suffices to show that for each $x \in X$, the real-valued function on $T$ by $t \rightarrow d(x, F(t))$ is measurable. For any $B, C \in C B(X)$, we have

$$
|d(x, B)-d(x, C)| \leqq D(B, C),
$$

thus

$$
\left|d\left(x, F_{n}(t)\right)-d(x, F(t))\right| \leqq D\left(F_{n}(t), F(t)\right) .
$$

Since for any $t \in T, D\left(F_{n}(t), F(t)\right) \rightarrow 0$ as $n \rightarrow \infty, d\left(x, F_{n}(t)\right) \rightarrow d(x, F(t))$ as $n \rightarrow \infty$. Therefore, $d(x, F(\cdot))$ is the pointwise limit of measurable functions $\left\{d\left(x, F_{n}(\cdot)\right)\right\}$, hence measurable.

Proposition 2. Let $F: T \times X \rightarrow C B(X)$ be a mapping such that for each $t \in T, F(t, \cdot)$ is $k(t)$-Lipschitz and for each $x \in X, F(\cdot, x)$ is measurable. Let $u: T \rightarrow X$ be a measurable mapping, then the mapping $G: T \rightarrow C B(X)$ defined by $G(t)=F(t, u(t))(t \in T)$ is measurable.

Proof. Since $X$ is separable, there exists a countable subset $\left\{x_{i}\right\}$ of $X$ such that $\operatorname{cl}\left(\left\{x_{i}\right\}\right)=X$, where $\operatorname{cl}(Y)$ is the closure of $Y$. For each $n$, denote

$$
B_{1 n}=\left\{x \in X: d\left(x, x_{1}\right) \leqq 1 / n\right\}
$$

and

$$
B_{\text {in }}=\left\{x \in X: d\left(x, x_{i}\right) \leqq 1 / n\right\}-\bigcup_{j=1}^{i-1} B_{j n} \quad(i=2,3, \cdots),
$$

then $\left\{B_{i n}\right\}$ is a countable measurable partition of $X$, that is, $B_{i n} \in \mathscr{B}, \quad \bigcup_{i=1}^{\infty} B_{i n}=X$ and if $i \neq j$, then $B_{i n} \cap B_{i n}=\varnothing$. Define $F_{n}: T \times X \rightarrow C B(X)$ as follows:

$$
F_{n}(t, x)=F\left(t, x_{\imath}\right) \quad \text { if } \quad t \in T, \quad x \in B_{\imath n} .
$$


Then for any open subset $B$ of $X$,

$$
\begin{aligned}
\left\{(t, x): F_{n}(t, x) \cap B \neq \varnothing\right\} & \\
= & \bigcup_{i=1}^{\infty}\left\{t \in T: F\left(t, x_{i}\right) \cap B \neq \varnothing\right\} \times B_{\text {in }} \in \mathscr{A} \times \mathscr{B},
\end{aligned}
$$

where $\mathscr{A} \times \mathscr{B}$ is the product $\sigma$-algebra on $T \times X$. Thus, for any $n, F_{n}$ is $\mathscr{A} \times \mathscr{B}$-measurable. For each $t \in T, x \in X$, there exists a unique $i$ such that $x \in B_{\text {in }}$ and

$$
\begin{aligned}
D\left(F_{n}(t, x), F(t, x)\right) & =D\left(F\left(t, x_{i}\right), F(t, x)\right) \\
& \leqq k(t) d\left(x_{i}, x\right) \leqq k(t) / n
\end{aligned}
$$

Hence $D\left(F_{n}(t, x), F(t, x)\right) \rightarrow 0$ as $n \rightarrow \infty$. By Proposition $1, F$ is $\mathscr{A} \times \mathscr{B}$ measurable. The mapping $g: T \rightarrow T \times X$ defined by $g(t)=(t, u(t))$ $(t \in T)$ is measurable in the sense that $g^{-1}(\mathscr{A} \times \mathscr{B}) \subset \mathscr{A}$. It follows that for any open subset $B$ of $X$,

$$
G^{-1}(B)=g^{-1}(\{(t, x): F(t, x) \cap B \neq \varnothing\}) \in \mathscr{A},
$$

and $G$ is measurable.

Proposition 3. Let $Y$ be a metric space, $f: T \times X \rightarrow Y$ a mapping such that for any $t \in T, f(t, \cdot)$ is continuous and for any $x \in X, f(\cdot, x)$ is measurable. Let $F: T \rightarrow 2^{X}$ be a measurable mapping such that for each $t \in T, F(t)$ is nonempty closed, and $U$ an open subset of $Y$. Then the mapping $G: T \rightarrow 2^{x}$ by $G(t)=\{x \in F(t): f(t, x) \in U\}(t \in T)$ is measurable.

Proof. By Himmelberg [5, Theorem 5.6], there exists a countable family $\left\{u_{n}\right\}$ of measurable selectors of $F$ such that for each $t \in T$, $\operatorname{cl}\left(\left\{u_{n}(t)\right\}\right)=F(t)$. Let $B$ be an open subset of $X$, then

$$
\begin{aligned}
G^{-1}(B) & =\{t \in T: f(t, x) \in U \text { for some } x \in F(t) \cap B\} \\
& =\left\{t \in T: f\left(t, u_{n}(t)\right) \in U, u_{n}(t) \in B \text { for some } n\right\} \\
& =\bigcup_{n=1}^{\infty}\left\{t \in T: f\left(t, u_{n}(t)\right) \in U\right\} \cap u_{n}^{-1}(B) .
\end{aligned}
$$

As in the proof of [5, Theorem 6.5],

$$
\left\{t \in T: f\left(t, u_{n}(t)\right) \in U\right\} \in \mathscr{A},
$$

hence $G^{-1}(B) \in \mathscr{A}$ and $G$ is measurable. 
Proposition 4. Let $F, G: T \rightarrow C B(X)$ be measurable mappings, $u: T \rightarrow X$ a measurable selector of $F, r: T \rightarrow(0, \infty)$ a measurable function. Then there exists a measurable selector $v: T \rightarrow X$ of $G$ such that for any $t \in T$,

$$
d(u(t), v(t)) \leqq D(F(t), G(t))+r(t)
$$

Proof. By Himmelberg [5, Theorem 5.6], there exist a countable family $\left\{u_{n}\right\}$ of measurable selectors of $F$ and a countable family $\left\{v_{n}\right\}$ of measurable selectors of $G$ such that for each $t \in T, \operatorname{cl}\left(\left\{u_{n}(t)\right\}\right)=F(t)$ and $\operatorname{cl}\left(\left\{v_{n}(t)\right\}\right)=G(t)$, respectively. It follows that

$$
D(F(t), G(t))=\max \left\{\sup _{i} \inf _{l} d\left(u_{i}(t), v_{l}(t)\right), \sup _{j} \inf _{i} d\left(u_{i}(t), v_{l}(t)\right)\right\},
$$

hence the real-valued function $D(F(\cdot), G(\cdot))$ on $T$ is measurable. Define mappings $f: T \times X \rightarrow R$ and $G_{1}: T \rightarrow 2^{X}$ by

$$
f(t, x)=d(u(t), x)-D(F(t), G(t))-r(t)
$$

and

$$
G_{1}(t)=\{x \in G(t): f(t, x)<0\}
$$

then by Proposition $3, G_{1}$ is measurable, and by definition of the Hausdorff metric, $G_{1}(t)$ is nonempty for all $t \in T$. Thus, the mapping $G_{2}: T \rightarrow C B(X)$ by $G_{2}(t)=\operatorname{cl}\left(G_{1}(t)\right)(t \in T)$ is measurable and has a measurable selector $v: T \rightarrow X$ by Kuratowski and Ryll-Nardzewski [6, Theorem, p. 398]. For this $v$, we have the desired conclusion.

4. A random fixed point theorem. Now we prove a random fixed point theorem for a multivalued contraction mapping.

THEOREM. Let $F: T \times X \rightarrow C B(X)$ be a mapping such that for each $x \in X, F(\cdot, x)$ is measurable and for each $t \in T, F(t, \cdot)$ is $k(t)$ contraction, where $k: T \rightarrow[0,1)$ is a measurable function. Then there exists a measurable mapping $u: T \rightarrow X$ such that for any $t \in T, u(t) \in$ $F(t, u(t))$.

Proof. Denote $A_{1}=\{t \in T: 0<k(t)\}$ and $A_{2}=T-A_{1}$, then $A_{1}, A_{2} \in \mathscr{A}$. We first consider on $A_{1}$. Take a measurable mapping $v_{0}: A_{1} \rightarrow X$. By Proposition 2, the mapping $F\left(\cdot, v_{0}(\cdot)\right): A_{1} \rightarrow C B(X)$ is measurable, hence there exists a measurable selector $v_{1}: A_{1} \rightarrow X$ of $F\left(\cdot, v_{0}(\cdot)\right)$ by Kuratowski and Ryll-Nardzewski [6]. Then by Proposi- 
tion 4 , there exists a measurable selector $v_{2}: A_{1} \rightarrow X$ of $F\left(\cdot, v_{1}(\cdot)\right)$ such that for any $t \in T$,

$$
d\left(v_{1}(t), v_{2}(t)\right) \leqq D\left(F\left(t, v_{0}(t)\right), F\left(t, v_{1}(t)\right)\right)+k(t) .
$$

By Proposition 4 again, there exists a measurable selector $v_{3}: A_{1} \rightarrow X$ of $F\left(\cdot, v_{2}(\cdot)\right)$ such that for any $t \in T$,

$$
d\left(v_{2}(t), v_{3}(t)\right) \leqq D\left(F\left(t, v_{1}(t)\right), F\left(t, v_{2}(t)\right)\right)+k(t)^{2} .
$$

By induction, we can choose a sequence of measurable mappings $v_{n}: A_{1} \rightarrow X$ such that for each $t \in T$,

$$
v_{n}(t) \in F\left(t, v_{n-1}(t)\right)
$$

and

$$
d\left(v_{n}(t), v_{n+1}(t)\right) \leqq D\left(F\left(t, v_{n-1}(t)\right), F\left(t, v_{n}(t)\right)\right)+k(t)^{n} \quad(n=1,2, \cdots) .
$$

Let $t \in A_{1}$ be arbitrarily fixed. For any $n$, we have

$$
\begin{aligned}
d\left(v_{n}(t), v_{n+1}(t)\right) \leqq & D\left(F\left(t, v_{n-1}(t)\right), F\left(t, v_{n}(t)\right)\right)+k(t)^{n} \\
\leqq & k(t) d\left(v_{n-1}(t), v_{n}(t)\right)+k(t)^{n} \\
\leqq & k(t)\left\{D\left(F\left(t, v_{n-2}(t)\right), F\left(t, v_{n-1}(t)\right)\right)+k(t)^{n-1}\right\}+k(t)^{n} \\
\leqq & k(t)^{2} d\left(v_{n-2}(t), v_{n-1}(t)\right)+2 k(t)^{n} \\
& --- \\
\leqq & k(t)^{n} d\left(v_{0}(t), v_{1}(t)\right)+n k(t)^{n}
\end{aligned}
$$

Thus, for every $n \leqq m$,

$$
\begin{aligned}
& d\left(v_{n}(t), v_{m+1}(t)\right) \leqq \sum_{i=n}^{m} d\left(v_{i}(t), v_{i+1}(t)\right) \\
& \leqq \sum_{i=n}^{m} k(t)^{i} d\left(v_{0}(t), v_{1}(t)\right)+\sum_{i=n}^{m} i k(t)^{i} .
\end{aligned}
$$

Since $0<k(t)<1,\left\{v_{n}(t)\right\}$ is a Cauchy sequence in $X$, hence converges to some $v(t) \in X$. It follows that for any $n$,

$$
\begin{aligned}
d(v(t), F(t, v(t)) & \leqq d\left(v(t), v_{n}(t)\right)+d\left(v_{n}(t), F(t, v(t))\right) \\
& \leqq d\left(v(t), v_{n}(t)\right)+D\left(F\left(t, v_{n-1}(t)\right), F(t, v(t))\right) \\
& \leqq d\left(v(t), v_{n}(t)\right)+k(t) d\left(v_{n-1}(t), v(t)\right)
\end{aligned}
$$


This implies that $d(v(t), F(t, v(t)))=0$. Since $F(t, v(t))$ is closed, $v(t) \in F(t, v(t))$. The mapping $v: A_{1} \rightarrow X$ is the pointwise limit of measurable mappings $\left\{v_{n}\right\}$, hence measurable. Now we consider on $A_{2}$. If $t \in A_{2}$, then for every $x, y \in X$,

$$
D(F(t, x), F(t, y)) \leqq k(t) d(x, y)=0 .
$$

Thus we can set $F(t, x)=F_{0}(t)$ for all $t \in A_{2}, \quad x \in X$, where $F_{0}: A_{2} \rightarrow C B(X)$ is measurable. By Kuratowski and Ryll-Nardzewski [6], there exists a measurable selector $w: A_{2} \rightarrow X$ of $F_{0}$. Then for any $t \in A_{2}, w(t) \in T(t, w(t))$. Define $u: T \rightarrow X$ by

$$
u(t)=\left\{\begin{array}{lll}
v(t) & \text { if } & t \in A_{1} \\
\text { or } & & \\
w(t) & \text { if } & t \in A_{2}
\end{array}\right.
$$

then $u$ is measurable and for each $t \in T, u(t) \in F(t, u(t))$.

\section{REFERENCES}

1. A. T. Bharucha-Reid, Random Integral Equations, Mathematics in Science and Engineering, Vol. 96, Academic Press, New York and London, 1972.

2. O. Hanš, Reduzierende zufällige Transformationen, Czechoslovak Math. J., 7 (1957), 154-158.

3. - Random operator equations, Proc. 4th Berkeley Symposium on Mathematical Statistics and Probability, Vol. II, Part I, 185-202, University of California Press, Berkeley, 1966.

4. F. Hiai, H. Umegaki, Integrals, conditional expectations and martingales of multivalued functions, J. Multivariate Anal., [to appear].

5. C. J. Himmelberg, Measurable relations, Fund. Math., 87 (1975), 53-72.

6. K. Kuratowski, C. Ryll-Nardzewski, A general theorem on selectors, Bull. Acad. Polon. Sci. Ser. Sci. Math. Astronom. Phys., 13 (1965), 397-403.

7. S. B. Nadler, Jr., Multi-valued contraction mappings, Pacific J. Math., 30 (1969), 475-488.

8. A. Špaček, Zufällige Gleichungen, Czechoslovak Math. J., 5 (1955), 462-466.

Received July 14, 1976.

TOKyo Institute of TECHNOLOGY

Oh-OKayama, Meguro-Ku

TOKYO 152 , JAPAN 



\section{Pacific Journal of Mathematics}

Vol. 68, No. 1

March, 1977

Richard Julian Bagby, On $L^{p}, L^{q}$ multipliers of Fourier transforms . .......

Robert Beauwens and Jean-Jacques Van Binnebeek, Convergence theorems in

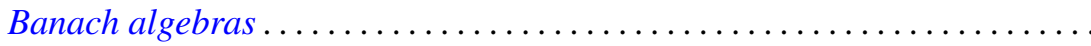

James Cyril Becker, Skew linear vector fields on spheres in the stable

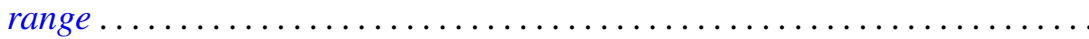

Michael James Beeson, Continuity and comprehension in intuitionistic formal

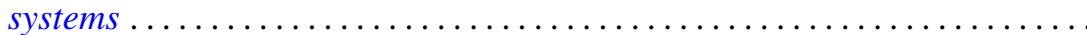

James K. Deveney, Generalized primitive elements for transcendental field

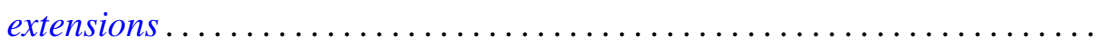

Samuel S. Feder, Samuel Carlos Gitler and K. Y. Lam, Composition properties

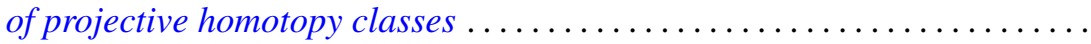

Nathan Jacob Fine, Tensor products of function rings under composition ......

Benno Fuchssteiner, Iterations and fixpoints . . . . . . . . . . . . . .

Wolfgang H. Heil, On punctured balls in manifolds

Shigeru Itoh, A random fixed point theorem for a multivalued contraction

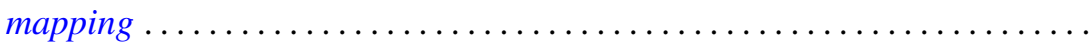

Nicolas P. Jewell, Continuity of module and higher derivations . . . . . . ......

Roger Dale Konyndyk, Residually central wreath products . . . . . . . . . . .

Linda M. Lesniak and John A. Roberts, On Ramsey theory and graphical

parameters.

Vo Thanh Liem, Some cellular subsets of the spheres.

Dieter Lutz, A perturbation theorem for spectral operators

P. H. Maserick, Moments of measures on convex bodies ... . . .

Stephen Joseph McAdam, Unmixed 2-dimensional local domains . .

D. B. McAlister and Norman R. Reilly, E-unitary covers for inverse semigroups...

William H. Meeks, III and Julie Patrusky, Representing codimension-one

homology classes by embedded submanifolds . . .

Premalata Mohapatro, Generalised quasi-Nörlund summability . .

Takahiko Nakazi, Superalgebras of weak-*Dirichlet algebras .

Catherine Louise Olsen, Norms of compact perturbations of operators .

William Henry Ruckle, Absolutely divergent series and isomorphism of

subspaces. II.

Bernard Russo, On the Hausdorff-Young theorem for integral operators .

Arthur Argyle Sagle and J. R. Schumi, Anti-commutative algebras and

homogeneous spaces with multiplications ............

Robert Evert Stong, Stiefel-Whitney classes of manifolds .

D. Suryanarayana, On a theorem of Apostol concerning Möbius functions of

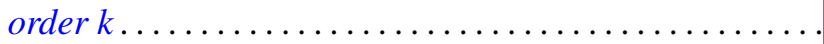

Yoshio Tanaka, On closedness of $C$ - and $C^{*}$-embeddings . . 